\title{
Avaliação da qualidade do atendimento da clínica-escola de Odontologia da UFCG
}

\section{na visão do usuário}

\author{
Assessment of the quality of attendance at the UFCG Dentistry school from the user's view \\ Evaluación de la cálida de asistencia en la escudela de Odontologia de UFCG desde la vista del \\ usuario
}

\author{
Luciana Ellen Dantas Costa \\ ORCID: https://orcid.org/0000-0003-4476-7900 \\ Universidade Federal de Campina Grande, Brasil \\ E-mail: ellendantascosta@yahoo.com.br \\ Rafael Alison Medeiros de Andrade \\ ORCID: https://orcid.org/0000-0002-8146-9796 \\ Universidade Federal de Campina Grande, Brasil \\ E-mail: sd.rafael.alison@hotmail.com \\ Aryanny Lourenna de Sousa \\ ORCID: https://orcid.org/0000-0003-1077-0917 \\ Universidade Federal de Campina Grande, Brasil \\ E-mail: aryannyl.sousa@gmail.com \\ Fabiana Larissa Santos de Medeiros \\ ORCID: https://orcid.org/0000-0002-3804-600X \\ Universidade Federal de Campina Grande, Brasil \\ E-mail: fabiana.serido@hotmail.com \\ Maria Cecília de Azevedo Araújo \\ ORCID: https://orcid.org/0000-0001-6121-6436 \\ Universidade Federal de Campina Grande, Brasil \\ E-mail: mceciazevedo@gmail.com \\ Ana Beatriz Costa Almeida \\ ORCID: https://orcid.org/0000-0003-2966-7709 \\ Universidade Federal do Rio Grande do Norte, Brasil \\ E-mail: anabeatrizca26@gmail.com \\ Faldryene de Sousa Queiroz Feitosa \\ ORCID: https://orcid.org/0000-0001-8531-871X \\ Universidade Federal de Campina Grande, Brasil \\ E-mail: falqueiroz@hotmail.com
}

\begin{abstract}
Resumo
Objetivo: Avaliar a satisfação dos usuários atendidos na clínica-escola de odontologia da Universidade Federal de Campina Grande, Patos/PB, Brasil. Métodos: Um estudo descritivo de corte transversal, com abordagem quantitativa, foi realizado partindo-se de uma amostra de conveniência, composta por 200 usuários. As entrevistas foram realizadas em sala de espera, utilizando um instrumento formulado a partir da agregação de questionários previamente validados: o Questionário de Avaliação da Qualidade dos Serviços de Saúde Bucal (QASSaB); de dados sócio-demográficos e sobre autopercepção de saúde bucal. Os dados foram registrados em um software e analisados por meio de estatística descritiva e inferência uni e bivariada. Resultados: Observou-se um perfil de usuários mulheres, com idade média de 37,43 anos, casados e com renda mensal familiar superior a 1 salário mínimo e escolaridade superior a 8 anos de estudo, que utilizam o serviço público de saúde, consideram boa sua saúde bucal e que não apresentaram dor de origem dentária, nos últimos 6 meses. Todas as dimensões de qualidade foram avaliadas positivamente pelos usuários, com alto grau de satisfação. Apenas o intervalo de marcação entre as consultas apresentou um número considerável de insatisfação. Conclusão: O serviço oferecido na clínica-escola vai além dos conhecimentos técnicos adquiridos pelos acadêmicos na universidade. A atuação generalista e humanista segue os princípios éticos no processo saúde doença e resolvem de forma satisfatória e com qualidade as principais necessidades de saúde bucal que acometem seus usuários.
\end{abstract}

Palavras-chave: Saúde pública; Acesso aos serviços de saúde; Satisfação do paciente.

\footnotetext{
Abstract

Objective: To assess the satisfaction of users attended at the dental school clinic at the Federal University of Campina Grande, Patos/PB, Brazil. Methods: A descriptive cross-sectional study, with a quantitative approach, was carried out starting from a convenience sample of 200 users. The interviews were conducted in a waiting room, using an instrument formulated from the aggregation of previously validated questionnaires: the Oral Health Services Quality Assessment
} 
Questionnaire (QASSaB); socio-demographic data and self-perception of oral health. Data were registered in a software and analyzed using descriptive statistics and uni and bivariate inference. Results: There was a profile of female users, with an average age of 37.43 years, married and with a monthly family income above 1 minimum wage and education over 8 years of study, who use the public health service, consider it good their oral health and that they did not present pain of dental origin, in the last 6 months. All dimensions of quality were positively evaluated by users, with a high degree of satisfaction. Only the appointment interval between appointments showed a considerable number of dissatisfaction. Conclusion: The service offered at the clinic-school goes beyond the technical knowledge acquired by academics at the university. The generalist and humanist action follows ethical principles in the health-disease process and satisfactorily and with quality solve the main oral health needs that affect its users.

Keywords: Public health; Health services accessibility; Patient satisfaction.

\section{Resumen}

Objetivo: Evaluar la satisfacción de los usuarios atendidos en la clínica de la escuela de odontología de la Universidad Federal de Campina Grande, Patos / PB, Brasil. Métodos: se realizó un estudio descriptivo transversal, con enfoque cuantitativo, a partir de una muestra de conveniencia de 200 usuarios. Las entrevistas se realizaron en la sala de espera, utilizando un instrumento formulado a partir de la agregación de cuestionarios previamente validados: el Cuestionario de Evaluación de la Calidad de los Servicios de Salud Bucal (QASSaB); datos sociodemográficos y autopercepción de la salud bucal. Los datos se registraron en un software y se analizaron mediante estadística descriptiva e inferencia uni y bivariada. Resultados: Se encontró un perfil de usuarias, con una edad promedio de 37,43 años, casadas y con un ingreso familiar mensual superior a 1 salario mínimo y educación superior a 8 años de estudio, que utilizan el servicio público de salud, consideran bueno su salud bucal. y que no se presentaron dolor de origen dental, en los últimos 6 meses. Todas las dimensiones de la calidad fueron evaluadas positivamente por los usuarios, con un alto grado de satisfacción. Solo el intervalo de citas entre citas mostró un número considerable de insatisfacción. Conclusión: El servicio que se ofrece en la clínica-escuela va más allá de los conocimientos técnicos adquiridos por los académicos de la universidad. La acción generalista y humanista sigue principios éticos en el proceso salud-enfermedad y soluciona satisfactoriamente y con calidad las principales necesidades de salud bucodental que afectan a sus usuarios.

Palavras clave: Salud pública; Accesibilidad a los servicios de salud; Satisfacción del paciente.

\section{Introdução}

Durante a formação do graduando em Odontologia, são realizadas em clínica, atividades que proporcionam um aprendizado eficiente tanto na dimensão técnica quanto na inter-relação profissional-paciente, além disso, pesquisas são realizadas nesse ambiente por ser o mais propício à mostrarem a real satisfação dos pacientes com relação ao atendimento prestado por parte dos alunos, professores e também pelos funcionários da clínica-escola (Sousa et al., 2015).

A avaliação dos pacientes sobre o atendimento odontológico prestado nas faculdades permite uma melhor compreensão dos fatores negativos e positivos gerados pelo atendimento prestado, podendo assim levar a reorganização de serviços, bem como a construção de ideias para uma melhoria do atendimento (Bordin et al., 2017), corrigindo problemas comuns na organização dos serviços de saúde e auxiliando na identificação das limitações dos serviços de acordo com o perfil do paciente, certificando assim melhorias quanto a atuação das práticas desenvolvidas (Azevedo \& Rocha, 2018). Dessa forma, gestores e trabalhadores podem verdadeiramente conhecer as reais necessidades dos usuários dos serviços de saúde e buscar continuamente processos de trabalhos que possam satisfazê-los (Souza et al., 2014).

Para avaliar a qualidade dos serviços de saúde, não se deve focar apenas no atendimento em si, mas considerar também as condições estruturais (recursos financeiros, humanos, físicos e organizacionais), ou seja, aquelas que proporcionam o universo de práticas a serem avaliadas, e podem interferir na opinião do usuário quanto ao atendimento clínico propriamente dito (Donabedian, 1990).

Os estudos, na sua maioria, avaliam a qualidade do atendimento em faculdades de Odontologia, sob a visão de alunos e professores, porém, a satisfação do usuário com os serviços de saúde é condição essencial para que se possa avaliar a qualidade do mesmo. A ótica do paciente sobre a qualidade do serviço ofertado vem, ao longo dos anos, se tornando uma preocupação das instituições que oferecem os serviços de saúde, sejam estas públicas ou privadas, o que, a partir de análises sobre a satisfação e/ou insatisfação dos receptores do serviço oferecido, estes podem ser aprimorados e melhorados, caso necessário (Torres \& Costa, 2015). 
O propósito deste estudo foi conhecer o perfil dos pacientes atendidos na clínica-escola de odontologia da UFCG assim como avaliar grau de satisfação com o atendimento ofertado, fornecendo subsídios teóricos para o planejamento dos serviços oferecidos à comunidade.

\section{Metodologia}

A pesquisa foi submetida a análise do Comitê de Ética em Pesquisa do Hospital Universitário Alcides Carneiro/ UFCG, obtendo parecer favorável de $\mathrm{n}^{\circ}$ 1.534.708. Sendo assegurada a confidencialidade dos dados coletados, do anonimato e da não utilização das informações em prejuízo dos envolvidos, como preconizam os documentos internacionais e a resolução CNS nº 466/2012.

Realizou-se um estudo descritivo de corte transversal, com abordagem quantitativa, partindo de uma amostra de conveniência, realizada na Clínica-escola de Odontologia da Universidade Federal de Campina Grande (UFCG), no Campus de Patos/PB. A amostra foi composta por 200 pacientes de ambos os sexos, atendidos na clínica-escola de Odontologia da UFCG no período de março a setembro de 2019, respeitando o universo total de clínicas em funcionamento neste período. Foram excluídos da pesquisa: pacientes menores de 18 anos, que tivessem sido submetidos a apenas um procedimento clínico anterior e os que recusaram assinar o termo de consentimento livre e esclarecido. A amostra foi selecionada de acordo com o cronograma de atendimento das clínicas, de forma que pacientes de todas as especialidades participassem do estudo, sendo em cada turno de atendimento incluídos, no máximo, 10 pacientes. Quando o número de pacientes na sala de espera excedeu essa quantidade, a escolha foi feita mediante sorteio.

Os dados foram coletados por meio de entrevistas, os usuários eram abordados na recepção e convidados, um por vez, a preencherem ao questionário em uma sala reservada de modo a minimizar os riscos de exposição e constrangimento. O instrumento de coleta foi formulado a partir da agregação de questionários previamente validados como o Questionário de Avaliação do Serviços de Saúde Bucal (QASSaB) e de dados sócio-demográficos, sobre a autopercepção de saúde bucal (Fernandes, 2002; Magalhães et al., 2015). O QASSaB baseia-se no modelo Donabedian composto por 24 questões que avaliam 10 dimensões da qualidade do atendimento, como: disponibilidade dos serviços; resolutividade; ambiente físico do serviçolimpeza; relações humanas (relações com outros profissionais de saúde); relações humanas (relação dentista/paciente); qualidade técnico-científica dos equipamentos; eficiência; eficácia; equidade e aceitabilidade (Fernandes, 2002; Magalhães et al., 2015; Oliveira et al., 2012; Brunhauser et al., 2013).

Os dados foram registrados na forma de banco de dados do programa de informática SPSS® (Statistical Package for the Social Sciences) versão 20.0, e analisados por meio de estatística descritiva e inferência uni e bivariada. Para os procedimentos descritivos, foram apresentados os dados brutos e relativos (frequências e porcentagens), ao passo que para os procedimentos de inferência estatística, utilizou-se o teste de associação entre as variáveis categóricas (teste do Qui-quadrado), adotando-se um nível de confiança de $95 \%$ e significância de 5\%. Na análise estatística, os dados com variáveis ordinais com mais de duas categorias foram reagrupadas para aumentar a eficiência dos testes. Foi construída uma variável dicotômica agrupando a categoria "muito satisfeito"com a "satisfeito" e a categoria "pouco satisfeito"com "insatisfeito", dessa forma, a variável dependente, obtida a partir das questões " Como você classificaria a forma como foi resolvido o problema que lhe trouxe ao serviço?" e "Levando em conta a qualidade dos serviços odontológicos executados e os recursos gastos por você com transporte e pagamento de taxas, mesmo assim, vc acha que valeu a pena?", ficaram com as categorias "muito satisfeito" e "pouco satisfeito". 


\section{Resultados}

A tabela 1 retrata o perfil dos 200 usuários do serviço da clínica escola de Odontologia da UFCG, avaliado através das perguntas referentes à características sócio-demográficas (sexo, idade, estado civil, renda mensal familiar, escolaridade e beneficiários de programas sociais), serviço de odontologia que utiliza, dor de dente nos últimos 6 meses e autopercepção de saúde bucal. Observa-se um perfil de usuários do sexo feminino (72,5\%), com idade média de 37,43 anos $\pm 12,33$ )(18 a 74 anos), casados (53,0\%), com renda mensal familiar acima de 1 salário mínimo (79,5\%), com segundo grau completo (33,0\%), não beneficiários de programa social $(65,0 \%)$, que utilizam o serviço público de saúde $(84,5 \%)$, sem experiência de dor nos últimos 6 meses $(66,5 \%)$ e que auto-consideram boa sua saúde bucal $(74,0 \%)$.

Tabela 1 - Frequência e percentual das variáveis independentes dos usuários da clínica-escola da UFCG, Patos, PB, 2019.

\begin{tabular}{llll}
\hline & & \multicolumn{2}{c}{ Total } \\
\cline { 2 - 4 } Variáveis Independentes & $\mathbf{N}$ & $\%$ \\
\hline
\end{tabular}

\section{Sexo}

Masculino

Idade

\section{Estado civil}

Casado

Viúvo

Renda Familiar mensal

$\leq 1 \mathrm{SM}$

$>1 \mathrm{SM}$

\section{Escolaridade}

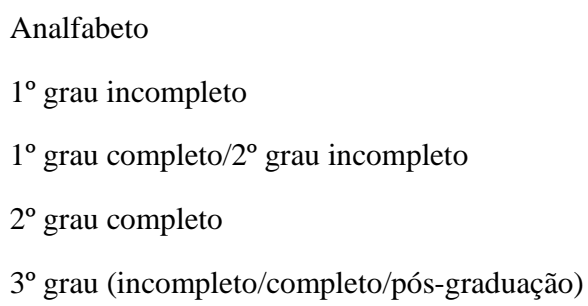

\section{Beneficiário de programa social}

\section{Serviço de odontologia que utiliza}


Privado

Dor de dente nos últimos 6 meses

Com dor

Sem dor

Não respondeu

\section{Autopercepção de saúde bucal}

Excelente

Boa

Ruim
13

39
6,5

Fonte: Autores.

A avaliação da clínica-escola da UFCG de acordo com as dimensões de qualidade pode ser visualizada na Tabela 2.

Tabela 2 - Avaliação da Clínica-escola da UFCG, segundo as dimensões da qualidade (QASSAB), Patos, PB, 2019.

\begin{tabular}{|c|c|c|c|c|c|c|}
\hline \multirow{2}{*}{$\begin{array}{l}\text { DIMENSÕES DE } \\
\text { QUALIDADE }\end{array}$} & \multicolumn{6}{|c|}{ Satisfação dos usuários } \\
\hline & $\mathbf{n}(\%)$ & $\mathbf{n}(\%)$ & n $(\%)$ & n $(\%)$ & n $(\%)$ & n $(\%)$ \\
\hline \multicolumn{7}{|c|}{ DIMENSÃO 1 - Disponibilidade dos serviços } \\
\hline Obtenção de vaga & $\begin{array}{c}\text { Não se aplica } \\
0(0,0)\end{array}$ & $\begin{array}{l}\text { Muito difícil } \\
3(1,5)\end{array}$ & $\begin{array}{c}\text { Difícil } \\
13(6,5)\end{array}$ & $\begin{array}{l}\text { Nem difícil } \\
\text { nem fácil } \\
39(19,5)\end{array}$ & $\begin{array}{c}\text { Fácil } \\
127(63,5)\end{array}$ & $\begin{array}{l}\text { Muito fácil } \\
18(9,0)\end{array}$ \\
\hline Intervalo entre consultas & $\begin{array}{c}\text { Não se aplica } \\
0(0,0)\end{array}$ & $\begin{array}{l}\text { Muito longo } \\
6(3,0)\end{array}$ & $\begin{array}{c}\text { Longo } \\
45(22,5)\end{array}$ & $\begin{array}{l}\text { Nem curto } \\
\text { nem longo } \\
81(40,5)\end{array}$ & $\begin{array}{c}\text { Curto } \\
63(31,5)\end{array}$ & $\begin{array}{l}\text { Muito curto } \\
5(2,5)\end{array}$ \\
\hline Tempo de espera & $\begin{array}{c}\text { Não se aplica } \\
0(0,0)\end{array}$ & $\begin{array}{l}\text { Muito longo } \\
1(5,0)\end{array}$ & $\begin{array}{l}\text { Longo } \\
20(10,0)\end{array}$ & $\begin{array}{l}\text { Nem curto } \\
\text { Nem longo } \\
79(39,5)\end{array}$ & $\begin{array}{c}\text { Curto } \\
93(46,5)\end{array}$ & $\begin{array}{c}\text { Muito curto } \\
7(3,5)\end{array}$ \\
\hline \multicolumn{7}{|l|}{ DIMENSÃO 2 - Resolutividade } \\
\hline Resolutividade do problema & $\begin{array}{c}\text { Não se aplica } \\
1(0,5)\end{array}$ & $\begin{array}{c}\text { Não resolvido } \\
25(12,5)\end{array}$ & $\begin{array}{c}\text { Mal resolvido } \\
2(1,0)\end{array}$ & $\begin{array}{c}\text { +/- resolvido } \\
49(24,5)\end{array}$ & $\begin{array}{c}\text { Resolvido } \\
95(47,5)\end{array}$ & $\begin{array}{c}\text { Muito bem resolvido } \\
28(14,0)\end{array}$ \\
\hline Aparência dos dentes tratados & $\begin{array}{l}\text { Não se aplica } \\
52(26,5)^{*}\end{array}$ & $\begin{array}{c}\text { Insatisfeito } \\
4(2,0)\end{array}$ & $\begin{array}{l}\text { Um pouco } \\
\text { satisfeito } \\
11(5,5)\end{array}$ & $\begin{array}{l}\text { Satisfeito } \\
87(43,5)\end{array}$ & $\begin{array}{c}\text { Muito satisfeito } \\
27(13,5)\end{array}$ & $\begin{array}{c}\text { Totalmente satisfeito } \\
19(9,5)\end{array}$ \\
\hline $\begin{array}{c}\text { Satisfeito com os dentes } \\
\text { posteriores - capacidade de } \\
\text { mastigação }\end{array}$ & $\begin{array}{l}\text { Não se aplica } \\
105(52,5)^{* *}\end{array}$ & $\begin{array}{c}\text { Insatisfeito } \\
2(1,0)\end{array}$ & $\begin{array}{l}\text { Um pouco } \\
\text { satisfeito } \\
8(4,0)\end{array}$ & $\begin{array}{l}\text { Satisfeito } \\
65(32,5)\end{array}$ & $\begin{array}{c}\text { Muito satisfeito } \\
9(4,5)\end{array}$ & $\begin{array}{c}\text { Totalmente satisfeito } \\
11(5,5)\end{array}$ \\
\hline \multicolumn{7}{|l|}{ DIMENSÃO 3 - Ambiente físico } \\
\hline $\begin{array}{c}\text { Limpeza da recepção/sala de } \\
\text { espera }\end{array}$ & $\begin{array}{l}\text { Não se aplica } \\
0(0,0)\end{array}$ & $\begin{array}{c}\text { Péssima } \\
0(0,0)\end{array}$ & $\begin{array}{l}\text { Ruim } \\
1(0,5)\end{array}$ & $\begin{array}{l}\text { Regular } \\
5(2,5)\end{array}$ & $\begin{array}{c}\text { Boa } \\
112(56,0)\end{array}$ & $\begin{array}{l}\text { Excelente } \\
82(41,0)\end{array}$ \\
\hline Limpeza da clínica & $\begin{array}{l}\text { Não se aplica } \\
0(0,0)\end{array}$ & $\begin{array}{l}\text { Péssima } \\
0(0,0)\end{array}$ & $\begin{array}{c}\text { Ruim } \\
0(0,0)\end{array}$ & $\begin{array}{l}\text { Regular } \\
5(2,5)\end{array}$ & $\begin{array}{c}\text { Boa } \\
108(54,0)\end{array}$ & $\begin{array}{l}\text { Excelente } \\
87(43,5)\end{array}$ \\
\hline $\begin{array}{c}\text { Como se sente em relação ao } \\
\text { conforto }\end{array}$ & $\begin{array}{l}\text { Não se aplica } \\
0(0,0)\end{array}$ & $\begin{array}{c}\text { Desconfortá- } \\
\text { vel } \\
1(0,5)\end{array}$ & $\begin{array}{l}\text { Um pouco } \\
\text { confortável } \\
4(2,0)\end{array}$ & $\begin{array}{l}\text { Confortável } \\
153(76,5)\end{array}$ & $\begin{array}{c}\text { Muito } \\
\text { confortável } \\
27(13,5)\end{array}$ & $\begin{array}{l}\text { Totalmente } \\
\text { Confortável } \\
15(7,5)\end{array}$ \\
\hline
\end{tabular}

DIMENSÕES 4 E 5 - Relações humanas (funcionários) / (relação com alunos e professores)

\begin{tabular}{|c|c|c|c|c|c|c|}
\hline $\begin{array}{l}\text { Atenção recebida dos } \\
\text { funcionários na recepção }\end{array}$ & $\begin{array}{l}\text { Não se aplica } \\
0(0,0)\end{array}$ & $\begin{array}{l}\text { Péssima } \\
0(0,0)\end{array}$ & $\begin{array}{l}\text { Ruim } \\
2(1,0)\end{array}$ & $\begin{array}{l}\text { Regular } \\
12(6,0)\end{array}$ & $\begin{array}{c}\text { Boa } \\
99(49,5)\end{array}$ & $\begin{array}{l}\text { Excelente } \\
87(43,5)\end{array}$ \\
\hline $\begin{array}{l}\text { Atenção recebida dos alunos e } \\
\text { professores }\end{array}$ & $\begin{array}{l}\text { Não se aplica } \\
0(0,0)\end{array}$ & $\begin{array}{c}\text { Péssima } \\
0(0,0)\end{array}$ & $\begin{array}{l}\text { Ruim } \\
0(0,0)\end{array}$ & $\begin{array}{l}\text { Regular } \\
2(1,0)\end{array}$ & $\begin{array}{c}\text { Boa } \\
57(28,5)\end{array}$ & $\begin{array}{l}\text { Excelente } \\
141(70,5)\end{array}$ \\
\hline
\end{tabular}




\begin{tabular}{|c|c|c|c|c|c|c|}
\hline $\begin{array}{c}\text { Confiança depositada nos } \\
\text { alunos }\end{array}$ & $\begin{array}{l}\text { Não se aplica } \\
0(0,0)\end{array}$ & $\begin{array}{l}\text { Péssima } \\
0(0,0)\end{array}$ & $\begin{array}{l}\text { Ruim } \\
0(0,0)\end{array}$ & $\begin{array}{l}\text { Regular } \\
22(11,0)\end{array}$ & $\begin{array}{c}\text { Boa } \\
84(42,0)\end{array}$ & $\begin{array}{l}\text { Excelente } \\
94(47,0)\end{array}$ \\
\hline \multicolumn{7}{|c|}{ DIMENSÃO 6 - Qualidade dos equipamentos } \\
\hline $\begin{array}{l}\text { Modernidade dos } \\
\text { equipamentos }\end{array}$ & $\begin{array}{l}\text { Não se aplica } \\
0(0,0)\end{array}$ & $\begin{array}{l}\text { Ultrapassados } \\
\quad 0(0,0)\end{array}$ & $\begin{array}{c}\text { Desatualiza-dos } \\
0(0,0)\end{array}$ & $\begin{array}{l}\text { Nem uma coisa } \\
\text { Nem outra } \\
27(13,5)\end{array}$ & $\begin{array}{l}\text { Modernos } \\
156(78,0)\end{array}$ & $\begin{array}{c}\text { Super } \\
\text { modernos } \\
17(8,5)\end{array}$ \\
\hline Conservação dos equipamentos & $\begin{array}{l}\text { Não se aplica } \\
0(0,0)\end{array}$ & $\begin{array}{l}\text { Péssima } \\
0(0,0)\end{array}$ & $\begin{array}{l}\text { Ruim } \\
1(0,5)\end{array}$ & $\begin{array}{l}\text { Regular } \\
18(9,0)\end{array}$ & $\begin{array}{c}\text { Boa } \\
130(65,0)\end{array}$ & $\begin{array}{l}\text { Excelente } \\
51(25,5)\end{array}$ \\
\hline \multicolumn{7}{|l|}{ DIMENSÃO 7 - Eficiência } \\
\hline Custo-Benefício & $\begin{array}{l}\text { Não se aplica } \\
1(0,5)\end{array}$ & $\begin{array}{c}\text { Não valeu a pena } \\
1(0,5)\end{array}$ & $\begin{array}{l}\text { Pouquíssimo } \\
1(0,5)\end{array}$ & $\begin{array}{l}\text { Mais ou menos } \\
22(11,0)\end{array}$ & $\begin{array}{c}\text { Muito } \\
136(68,0)\end{array}$ & $\begin{array}{l}\text { Até demais } \\
39(19,5)\end{array}$ \\
\hline \multicolumn{7}{|l|}{ DIMENSÃO 8 - Eficácia } \\
\hline Frequência de uso de EPI & $\begin{array}{l}\text { Não se aplica } \\
0(0,0)\end{array}$ & $\begin{array}{l}\text { Nunca } \\
0(0,0)\end{array}$ & $\begin{array}{c}\text { Às vezes } \\
0(0,0)\end{array}$ & $\begin{array}{c}\text { Não me lembro } \\
0(0,0)\end{array}$ & $\begin{array}{l}\text { Quase sempre } \\
1(0,5)\end{array}$ & $\begin{array}{c}\text { Sempre } \\
199(99,5)\end{array}$ \\
\hline $\begin{array}{c}\text { Desconforto durante a } \\
\text { extração }\end{array}$ & $\begin{array}{l}\text { Não se aplica } \\
121(60,5)^{*}\end{array}$ & $\begin{array}{c}\text { Senti total } \\
\text { desconforto } \\
1(0,5)\end{array}$ & $\begin{array}{l}\text { Senti muito } \\
\text { desconforto } \\
2(1,0)\end{array}$ & $\begin{array}{l}\text { Senti desconforto } \\
9(4,5)\end{array}$ & $\begin{array}{l}\text { Senti muito } \\
\text { pouco } \\
\text { desconforto } \\
18(9,0)\end{array}$ & $\begin{array}{l}\text { Não senti nada } \\
\quad 49(24,5)\end{array}$ \\
\hline $\begin{array}{l}\text { Desconforto durante } \\
\text { restauração ou raspagem } \\
\text { gengival }\end{array}$ & $\begin{array}{l}\text { Não se aplica } \\
49(24,5)^{* *}\end{array}$ & $\begin{array}{l}\text { Senti total } \\
\text { desconforto } \\
1(0,5)\end{array}$ & $\begin{array}{l}\text { Senti muito } \\
\text { desconforto } \\
5(2,5)\end{array}$ & $\begin{array}{c}\text { Senti desconforto } \\
19(9,5)\end{array}$ & $\begin{array}{l}\text { Senti muito } \\
\text { pouco } \\
\text { desconforto } \\
32(16,0)\end{array}$ & $\begin{array}{l}\text { Não senti nada } \\
\quad 94(47,0)\end{array}$ \\
\hline $\begin{array}{l}\text { Restauração alta ou } \\
\text { arranhando }\end{array}$ & $\begin{array}{l}\text { Não se aplica } \\
95(47,5)^{* * *}\end{array}$ & $\begin{array}{l}\text { Senti total } \\
\text { desconforto } \\
0(0,0)\end{array}$ & $\begin{array}{l}\text { Senti muito } \\
\text { desconforto } \\
0(0,0)\end{array}$ & $\begin{array}{l}\text { Senti desconforto } \\
5(2,5)\end{array}$ & $\begin{array}{c}\text { Senti muito } \\
\text { pouco } \\
\text { desconforto } \\
12(6,0)\end{array}$ & $\begin{array}{l}\text { Não senti nada } \\
\quad 88(44,0)\end{array}$ \\
\hline Orientação de saúde bucal & $\begin{array}{l}\text { Não se aplica } \\
0(0,0)\end{array}$ & $\begin{array}{c}\text { Nunca } \\
26(13,0)\end{array}$ & $\begin{array}{l}\text { Raramente } \\
4(2,0)\end{array}$ & $\begin{array}{l}\text { Às vezes } \\
27(13,5)\end{array}$ & $\begin{array}{l}\text { Quase sempre } \\
29(14,5)\end{array}$ & $\begin{array}{c}\text { Sempre } \\
114(57,0)\end{array}$ \\
\hline \multicolumn{7}{|l|}{ DIMENSÃO 9 - Equidade } \\
\hline $\begin{array}{l}\text { Necessidades atendidas: } \\
\text { público x privado }\end{array}$ & $\begin{array}{l}\text { Não se aplica } \\
\quad 0(0,0)\end{array}$ & $\begin{array}{l}\text { Nunca são } \\
\text { atendidas } \\
0(0,0)\end{array}$ & $\begin{array}{l}\text { Diferente-mente } \\
\text { atendidas } \\
1(0,5)\end{array}$ & $\begin{array}{l}\text { Não me lembro } \\
5(2,5)\end{array}$ & $\begin{array}{l}\text { Parcialmen-te } \\
\text { atendidas } \\
3(1,5)\end{array}$ & $\begin{array}{l}\text { Igualmente atendidas } \\
191(95,5)\end{array}$ \\
\hline \multicolumn{7}{|l|}{ DIMENSÃO 10 - Aceitabilidade } \\
\hline $\begin{array}{c}\text { Explicação sobre o tratamento } \\
\text { mais adequado }\end{array}$ & $\begin{array}{l}\text { Não se aplica } \\
\quad 0(0,0)\end{array}$ & $\begin{array}{c}\text { Nunca me explica } \\
\text { nada } \\
10(5,0)\end{array}$ & $\begin{array}{c}\text { Raras vezes me } \\
\text { explica } \\
4(2,0)\end{array}$ & $\begin{array}{l}\text { Não me lembro } \\
12(6,0)\end{array}$ & $\begin{array}{l}\text { Na maioria das } \\
\text { vezes me } \\
\text { explica } \\
39(19,5)\end{array}$ & $\begin{array}{c}\text { Sempre me explica } \\
\text { tudo } \\
135(67,5)\end{array}$ \\
\hline $\begin{array}{l}\text { Opinião sobre melhor dia e } \\
\text { horário para ser atendido }\end{array}$ & $\begin{array}{l}\text { Não se aplica } \\
0(0,0)\end{array}$ & $\begin{array}{l}\text { Nunca pede a } \\
\text { minha opinião } \\
\quad 46(23,0)\end{array}$ & $\begin{array}{c}\text { Raras vezes pede } \\
\text { a minha opinião } \\
8(4,0)\end{array}$ & $\begin{array}{l}\text { Não me lembro } \\
11(5,5)\end{array}$ & $\begin{array}{l}\text { Na maioria das } \\
\text { vezes pede } \\
\text { minha opinião } \\
42(21,0)\end{array}$ & $\begin{array}{c}\text { Sempre pede a minha } \\
\text { opinião } \\
93(46,5)\end{array}$ \\
\hline
\end{tabular}

*Pacientes que realizaram apenas exodontias; **Pacientes que realizaram procedimentos que não interferissem na capacidade de mastigação; Fonte: Autores.

Na dimensão disponibilidade dos serviços de saúde bucal, a obtenção de uma vaga é considerado "fácil" pela maioria dos usuários (63,5\%), sendo indiferente, ou seja, nem curto nem longo, o intervalo entre as consultas (40,5\%), e curto o tempo de espera entre a chegada ao serviço e o momento do atendimento (46,5\%).

$\mathrm{Na}$ dimensão resolutividade, a maioria relatou que o problema que os trouxe ao serviço foi resolvido $(47,5 \%)$, que estavam satisfeitos com a aparência dos dentes tratados $(43,5 \%)$ e com a capacidade de mastigação dos dentes posteriores tratados $(32,5 \%)$.

Na dimensão ambiente físico, a maioria dos usuários classificou como boa a limpeza da recepção/sala de espera (56,0\%), a limpeza da clínica $(54,0 \%)$ e dos banheiros $(35,5 \%)$. No quesito conforto, 76,5\% disseram se sentir "confortável" na clínicaescola. 
Nas dimensões relações humanas funcionários e profissionais (professores e alunos), foi boa a atenção recebida dos funcionários na recepção (49,5\%), e excelente a atenção recebida dos alunos e professores (70,5\%), considerando como excelente o grau de confiança no aluno $(47,0 \%)$.

Os equipamentos da clínica-escola foram considerados modernos $(78,0 \%)$ e em bom estado de conservação e manutenção $(65,0 \%)$.

Ao analisar a eficiência do serviço, ou seja, a relação custo-benefício, 68,0\% dos usuários disseram ter valido muito a pena realizar o tratamento na clínica-escola, sob o ponto de vista da qualidade dos serviços odontológicos prestados.

Avaliando a dimensão eficácia, 99,5\% dos usuários afirmaram que os alunos sempre usavam equipamentos de proteção individual (EPI's), que incluem gorro, luvas, máscara, óculos e roupas limpas. Indagados sobre desconforto do tipo dor e pressão exagerada através das mãos dos alunos, para a maioria não houve qualquer desconfortos durante os procedimentos de extrações $(24,5 \%)$ e em restaurações ou raspagens gengivais (47,9\%), assim como de restauração alta ou arranhando (44,0\%). Ainda, os usuários relataram sempre receberam orientações de saúde bucal $(57,0 \%)$.

Ao avaliar tal dimensão de equidade, quase que a totalidade dos usuários tiveram suas necessidades igualmente atendidas quando comparadas às necessidades das pessoas de uma classe social mais alta $(95,5 \%)$.

$\mathrm{Na}$ dimensão aceitabilidade, os pacientes sempre recebiam explicações sobre o tratamento mais adequado (67,5\%), assim como questionados sobre o melhor dia e horário para o atendimento (46,5\%).

De um modo geral, após a avaliação das dimensões de qualidade por usuário, 187 (93,5\%) pacientes relataram estar muito/totalmente satisfeito com a qualidade do atendimento ofertado, enquanto que apenas 13 (6,5\%) mostraram-se insatisfeitos/pouco satisfeitos.

$\mathrm{Na}$ análise bivariada, apenas as variáveis independentes idade e autopercepção de saúde bucal foram significativamente associadas com a satisfação geral dos usuários na dimensão custo-benefício, sendo essa maior em indivíduos com idade superior a $33 \operatorname{anos}(p=0,004)$ e que tinham uma autopercepção ruim da saúde bucal ( $p=0,048)$ (Tabela 3$)$.

Tabela 3 - Avaliação do grau de satisfação dos usuários nas dimensões resolutividade do problema e relação custo-benefício associados às variáveis independentes, Patos, PB, 2019.

\begin{tabular}{|c|c|c|c|c|c|c|c|c|}
\hline \multicolumn{9}{|c|}{ SATISFAÇÃO DO USUÁRIO } \\
\hline & \multicolumn{4}{|c|}{ RESOLUTIVIDADE DO PROBLEMA } & \multicolumn{4}{|c|}{ RELAÇÃO CUSTO-BENEFÍCIO } \\
\hline & $\begin{array}{c}\text { Pouco } \\
\text { satisfeito }\end{array}$ & $\begin{array}{c}\text { Muito } \\
\text { satisfeito }\end{array}$ & Total & & $\begin{array}{c}\text { Pouco } \\
\text { satisfeito }\end{array}$ & $\begin{array}{c}\text { Muito } \\
\text { satisfeito }\end{array}$ & Total & \\
\hline $\begin{array}{l}\text { Variáveis } \\
\text { independentes }\end{array}$ & $\mathbf{N}(\%)$ & $\%$ & $\mathbf{N}$ & Valor de $\mathrm{p}$ & $\mathrm{N}(\%)$ & $\mathbf{N}(\%)$ & $\mathbf{N}$ & Valor de $\mathrm{p}$ \\
\hline \multicolumn{9}{|l|}{ Sexo } \\
\hline Masculino & $18(32,7)$ & $37(67,3)$ & 55 & 0,372 & $6(10,9)$ & $49(89,1)$ & 55 & 0,770 \\
\hline Feminino & $57(39,6)$ & $87(60,4)$ & 144 & & $18(12,4)$ & $127(87,6 \%)$ & 144 & \\
\hline \multicolumn{9}{|l|}{ Idade } \\
\hline$\leq .33$ anos & $34(43,0)$ & $45(57,0)$ & 79 & 0,206 & $16(20,0)$ & $64(80,0)$ & 80 & $0,004 *$ \\
\hline$>33$ anos & $41(34,2)$ & $79(65,8)$ & 120 & & $8(6,7)$ & $112(93,3)$ & 120 & \\
\hline Escolaridade (a & de estudo & & & & & & & \\
\hline
\end{tabular}




\begin{tabular}{|c|c|c|c|c|c|c|c|c|}
\hline$<8$ anos & $26(40,0)$ & $39(60,0)$ & 65 & 0,639 & $6(9,2)$ & $59(90,8)$ & 65 & 0,403 \\
\hline$>8$ anos de estudo & $49(36,6)$ & $85(63,4)$ & 134 & & $18(13,3)$ & $117(86,7)$ & 135 & \\
\hline \multicolumn{9}{|l|}{ Renda mensal } \\
\hline$<1 \mathrm{SM}$ & $14(34,1)$ & $27(65,9)$ & 41 & 0,599 & $7(17,1)$ & $34(82,9)$ & 41 & 0,262 \\
\hline$>2 \mathrm{SM}$ & $61(38,6)$ & $97(61,4)$ & 158 & & $17(10,7)$ & $142(89,3)$ & 159 & \\
\hline \multicolumn{9}{|c|}{ Beneficiário de programa social } \\
\hline Sim & $32(45,7)$ & $38(54,3)$ & 70 & 0,085 & $8(11,4)$ & $62(88,6)$ & 70 & 0,855 \\
\hline Não & $43(33,3)$ & $86(66,7)$ & 129 & & $16(12,3)$ & $114(87,7)$ & 130 & \\
\hline \multicolumn{9}{|l|}{ Tipo de serviço } \\
\hline Público & $66(39,3)$ & $102(60,7)$ & 168 & 0,279 & $19(11,2)$ & $150(88,8)$ & 169 & 0,442 \\
\hline Privado & $9(29,0)$ & $22(71,0)$ & 31 & & $5(16,1)$ & $26(83,9)$ & 31 & \\
\hline \multicolumn{9}{|c|}{ Dor nos últimos 6 meses } \\
\hline Sim & $29(44,6)$ & $36(55,4)$ & 65 & 0,184 & $8(12,5)$ & $57(87,7)$ & 65 & 0,955 \\
\hline Não & $46(34,8)$ & $86(65,2)$ & 132 & & $16(12,0)$ & $117(88,0)$ & 133 & \\
\hline \multicolumn{9}{|c|}{ Autopercepção de saúde bucal } \\
\hline Excelente/boa & $56(34,8)$ & $105(65,2)$ & 161 & 0,082 & $23(14,2)$ & $139(85,8)$ & 162 & $0,048^{*}$ \\
\hline Ruim & $19(50,0)$ & $19(50,0)$ & 38 & & $1(2,6)$ & $37(97,4)$ & 38 & \\
\hline
\end{tabular}

Fonte: Autores.

\section{Discussão}

A realização deste estudo possibilitou avaliar a qualidade do atendimento prestado na clínica-escola de odontologia da UFCG em todas as dimensões propostas por Fernandes (2002), baseado no modelo Donabediano de avaliação da qualidade, bem como permitiu conhecer o perfil sócio-demográfico e a autopercepção em saúde bucal dos usuários do serviço. O conhecimento destas variáveis fornecerá subsídios teóricos para o planejamento do atendimento e monitoramento das ações sobre a principal ótica, a dos usuários do serviço, uma vez que a satisfação do paciente é um propósito de grande importância a ser alcançado pelos serviços de saúde, o que possibilita um serviço resolutivo e de qualidade, de acordo com a necessidade local da comunidade assistida (Torres \& Costa, 2015). A identificação do perfil desses usuários, por sua vez, possibilita fornecer um atendimento individualizado de acordo com a realidade que o paciente se encontra, otimizando seu prognóstico (Sousa et al., 2015).

No presente estudo observou-se que a maioria dos usuários eram do sexo feminino, corroborando aos achados de Torres e Costa (2015); Borges et al., (2015), Kitamura et al., (2016) e Nascimento et al., (2020). Na maioria dos estudos observou-se uma maior procura por atendimento pelo sexo feminino possivelmente pela disponibilidade de horário, uma vez que a maioria das clínicas-escolas funciona em período diurno, assim como pelo comportamento mais preventivo e de autocuidado, uma vez que as mulheres se preocupam mais com a aparência e saúde que os homens.

A idade revelou predominância de indivíduos pertencentes ao grupo de adultos entre 34 e 60 anos, corroborando aos estudos Torres e Costa (2015) e Nascimento et al., (2020). Outros estudos mostraram resultados diferentes, como o de Mialhe et al. (2008) e Praxedes et al., (2017) que relataram maior procura pelo serviço de pacientes jovens. Diante, cabe destacar a 
importância da clínica-escola para os adultos, que além de necessitarem de um atendimento mais rápido e de qualidade, buscam a resolutividade dos problemas de maior prevalência para essa faixa etária, nas áreas de periodontia e reabilitação oral, esta última apresentando uma demanda reprimida significativamente alta no serviço público de saúde de Patos/PB, chegando a esperar até 6 meses para o primeiro atendimento.

Quanto a escolaridade, a grande maioria era alfabetizada, com instrução de nível médio completo semelhante aos resultados encontrados por Nascimento et al., (2020) e Souza et al., (2014). A maioria dos usuários apresentaram renda mensal familiar entre 1 e 3 salários mínimos, a mesma condição foi observada no estudo de Borges et al., (2015), não beneficiários de programa social e assistidos pelo setor público de saúde.

A partir destes dados é possível identificar a clínica-escola de odontologia da UFCG como um referencial de atendimento gratuito que presta serviço de qualidade, serviços estes que muitas vezes não estão disponíveis na rede pública de saúde, refletindo a importância da universidade para a cidade e a população da região na qual está inserida. Pêgo et al. (2016) ressaltaram a questão financeira dos entrevistados como um determinante para o tratamento odontológico de qualidade e vislumbram na Universidade uma oportunidade de realizá-lo gratuitamente. O que se tem observado é um aumento na procura pelo serviço das pessoas com alto grau de instrução, demonstrando interesse com a prevenção e tratamento das doenças bucais em instituições de ensino por oferecerem um serviço de qualidade, o que também observado por Nascimento e colaboradores na sua pesquisa (2020).

A dor de origem dentária nos últimos seis meses não foi relatada pela maioria dos usuários. Os mesmos autoconsideraram sua saúde bucal como boa. A autopercepção da saúde bucal é um importante indicativo de análise de saúde da população, uma vez que por meio dela o usuário tem consciência sobre a busca por tratamento e se empenha para que o tratamento seja resolvido (Magalhães et al., 2015; Santos et al., 2016).

Em relação às dimensões que avaliaram o grau de satisfação dos usuários atendidos, a clínica-escola de Odontologia da UFCG foi avaliada positivamente, corrobando aos resultados obtidos por Souza et al., (2014), Borges et al., (2015), Marafon (2017) e Nascimento et al., (2020).

No entanto, quando comparado aos estudos que avaliaram a qualidade do serviço odontológico em estabelecimentos públicos de saúde como UBS's e CEO's, verificou-se que há um padrão de insatisfação, principalmente quanto a disponibilidade dos serviços (Kitamura, 2016, Magalhaes et al., 2015).

A dimensão de disponibilidade dos serviços, é uma das mais importantes a serem avaliadas, principalmente quanto à obtenção de vaga, por ser condição para o uso. No presente estudo, os usuários demonstraram em sua maioria satisfação quanto a obtenção das vagas e tempo de espera pelo atendimento, resultado esse considerado positivo uma vez que é comum observar usuários insatisfeitos quanto à essas variáveis (Brás et al., 2015; Magalhães et al., 2015; Marafon, 2017; Torres \& Costa, 2015). No entanto, apesar de bem avaliado pela maioria dos usuários, o intervalo entre as consultas apresentou uma porcentagem negativa significativa no presente estudo, o que também foi relatado por Magalhães et al., (2015), evidenciando a necessidade de implementação de um sistema de retorno eficiente, simples e prático, com intervalos regulares.

Moimaz et al., (2017) afirmaram que o descontentamento no quesito "fila de espera" é rotineiro nos serviços de saúde, e é considerado o maior motivo de desistência dos usuários. A espera é um obstáculo para a utilização do serviço que, além de causar insatisfação, pode piorar o estado de saúde e prolongar o sofrimento e ansiedade do paciente.

Na dimensão resolutividade, os dados do presente estudo sugerem que os serviços estão de fato sendo resolutivos, estão respondendo as necessidades de saúde bucal dos seus usuários e estes estão satisfeitos. Borges et al., (2014), Nascimento et al., (2020), Souza et al., (2014) e Torres e Costa (2015) também observaram alto nível de satisfação dos usuários atendidos nas clínicas do departamento de odontologia da UFRN, Universidade Federal de Pernambuco, da Universidade Federal de 
Uberlândia e da Universidade de Itaúna - MG, respectivamente, evidenciando que, em geral, essa dimensão é bem avaliada pelos usuários atendidos em instituições de nível superior.

O ambiente odontológico também foi contemplado na avaliação. Os usuários avaliaram quanto à limpeza dos ambientes da recepção, sala de espera e clínicas da UFCG e quanto ao conforto, os quais foram considerados excelentes em sua maioria. Os equipamentos por sua vez, também foram considerados modernos. Nascimento et al., (2020) observaram em seu trabalho na Universidade Federal de Pernambuco que a organização da clínica mostrou associação significativa com a satisfação do usuário, e que tais considerações podem ter proporcionado uma sensação de acolhimento e valorização, tornando-os mais motivados, receptivos e colaboradores, melhorando a qualidade do atendimento. A organização e qualidade técnica-cientifica dos equipamentos de um serviço de saúde se faz necessário e importante, e pode exercer um resultado positivo sobre a seriedade e competência do trabalho realizado pela equipe sob a ótica do paciente (Souza et al., 2014).

A dimensão relações humanas, vista de forma positiva em sua totalidade, mostra o perfil humanizado apresentado pelos profissionais e alunos que compõem a clínica de odontologia da UFCG, resultado esse fundamental e esperado para os serviços de saúde em instituições de ensino, principalmente nessa nova fase de implementação das novas Diretrizes Curriculares Nacionais, que orientam como perfil de egresso do graduando em odontologia um profissional humanístico e ético, atento a dignidade da pessoa humana e às necessidades individuais e coletivas, que deve promover a saúde integral e transformar a realidade em benefício da sociedade (Brasil, 2021).

Uma das maiores dificuldades em estruturar as relações humanas encontra-se na formação dos profissionais de saúde. Segundo Rezende et al., (2015), as pessoas não só desejam profissionais capacitados tecnicamente, mas também almejam o aspecto humano nas relações pessoais, relacionados a educação, cordialidade, gentileza e comunicabilidade, além da simpatia, sendo essencial dar um significado mais humanístico à prática odontológica, pois, quanto mais o profissional compreende as pessoas que o procuram, mais positivo poderá tornar-se o tratamento. Os alunos/professores também foram avaliados positivamente quanto a explicação sobre o tratamento mais adequado para o paciente no presente estudo. Talvez o grau de confiança que os usuários depositaram nos alunos seja reflexo dessa relação humana positiva.

Na dimensão da eficiência, para a maioria dos entrevistados o custo-benefício "valeu muito a pena" ou "até demais" em realizar o tratamento na UFCG, mesmo diante de algumas barreiras/ dificuldades para acessar o serviço, corroborando aos achados de Torres \& Costa (2015). Essa dimensão foi selecionada para análise bivariada juntamente com a resolutividade do problema uma vez que constituem em importantes indicadores, por avaliar se tais serviços são eficientes e resolutivos, assim como sugerem a importância e o valor que esses serviços têm para a população no processo de cuidado em saúde (Magalhães et al., 2015). Na análise bivariada no presente estudo, as variáveis "idade" e "autopercepção de saúde bucal" foram significativamente associadas com a satisfação dos usuários, sendo maior nos usuários com idade superior a 33 anos e com autopercepção ruim de saúde bucal, o que pode estar relacionado a qualidade do serviço recebido na clínica-escola, ambiente este com equipamentos modernos, insumos e materiais odontológicos de qualidade, assim como profissionais especializados que ofertaram um tratamento resolutivo, o que difere muitas vezes do serviço publico que saúde do município uma vez que nem todos os serviços ofertados são eficientes e resolutos. Mialhe et al., (2008) associaram a satisfação do usuário a três fatores, o tratamento estar sendo supervisionado por um profissional muito bem titulado, a falta de condições para contratar um serviço particular e a inexistência da oferta desse serviço com a mesma qualidade no âmbito público.

Na dimensão eficácia, a frequência constante do uso de equipamento de proteção individual foi relatado por quase a totalidade dos usuários do presente estudo. Esses equipamentos são indispensáveis para a realização do atendimento, evitando ao máximo qualquer forma de contaminação, tanto do paciente, como do profissional que o atendeu (Borges et al., 2015). Resultados semelhantes foram encontrados também no estudo de Sousa et al., (2015) e Torres e Costa (2015). 
Ainda nessa dimensão, observou-se que parte dos usuários referiram algum tipo de desconforto tanto nas exodontias quanto nas restaurações/raspagens. Esses achados são comuns em estudos de avaliação de atendimento em saúde bucal, uma vez que o desconforto pode estar associado ao medo, quer seja do barulho do motor, da anestesia ou mesmo da extração, sendo esse, o maior obstáculo frente ao tratamento. O próprio consultório odontológico para muitos é considerado como um ambiente de natureza ansiogênica, muitas vezes os usuários ao adentrarem no serviço apresentam comportamentos de inquietação, palidez, midríase, transpiração excessiva, entre outros, e estando associado ao medo do tratamento, ele pode somatizar as sensações de desconforto e dor e assim, comprometer o atendimento odontológico (Melonardino et al., 2016; Martins et al., 2017). Dessa forma, uma boa relação profissional-paciente é necessária, pois muitas vezes é capaz de controlar e atenuar o medo presente em alguns pacientes.

No entanto, apesar de grande parte dos usuários avaliarem bem as dimensões de qualidade do atendimento em saúde bucal no presente estudo, nem todos foram informados sobre como evitar os problemas bucais, apresentando uma significativa porcentagem de usuários que nunca receberam orientações de saúde bucal, ou às receberam pouco ou raramente, o que mostra a necessidade de inserção e reforço de ações de promoção e prevenção, primordiais no cuidado integral da saúde da pessoa humana.

Outro achado importante diz respeito à dimensão da equidade no atendimento recebido, pois quase que em sua totalidade os usuários afirmaram que as necessidades de saúde bucal foram igualmente atendidas ao compararem os serviços públicos com os privados. Resultado semelhante foi observado no estudo de Torres e Costa (2015) que ao avaliarem o discurso coletivo dos usuários da clínica-escola da UFRN na categoria “Economia com qualidade” observaram os seguintes relatos: “Aqui fiz todo o tratamento, de graça e com qualidade. Além do benefício financeiro, fui bem tratado, num ambiente limpo, tranquilo e com alunos bem preparados". Os resultados do presente estudo podem ser considerados positivos e de grande importância uma vez que culturalmente e, principalmente, por influência das redes social e influenciadores digitais, que cada vez mais giram em torno de interesses no capital, os serviços ofertados em instituições de ensino por serem gratuitos são vistos como sem qualidade e aviltados, já o particular é enaltecido e supervalorizado, lhes trazendo status social (Magalhães et al., 2015, Nascimento et al., 2020).

Quanto a amostra do estudo, em média, antes do período da pandemia da COVID-19, 80 usuários eram atendidos semanalmente na clínica-escola entre primeira consulta e consultas de retorno. A amostra ficou restrita a 200 pacientes, pois durante os sete meses de coleta de dados alguns imprevistos atrapalharam o processo como: a falta de material odontológico, problemas estruturais e período de recesso acadêmico, impossibilitando um número maior em tempo hábil. No entanto, essa amostra foi superior aos estudos de Torres e Costa (2015) e Nascimento et al (2020) que entrevistaram 71 e 81 usuários, respectivamente.

A maioria dos estudos que avaliaram a qualidade de satisfação do usuário utilizaram o instrumento QASSAB, demonstrando que a maioria dos usuários estavam satisfeitos com o serviço prestado, satisfação que esteve associada a resolutividade do problema de saúde bucal que os motivou a procurar o serviço. É necessário pontuar os limites inerentes de estudos baseados na percepção do usuário, que por se tratar de uma avaliação subjetiva pode trazer implicações para esse tipo de avaliação. Segundo Magalhães et al. 2015, os pacientes podem diminuir suas expectativas quanto ao serviço já que não pagam pelo mesmo ou então avaliam positivamente por terem uma baixa capacidade crítica, assim como podem avaliar negativamente nos casos daqueles pacientes com alto grau de exigência. Outro tipo de viés é que esse estudo pode produzir é que em contextos desiguais da população, o simples fato de ser atendido e resolver o seu problema, ja torna os usuários satisfeitos.

Ressalta-se a importância desse estudo uma vez que não há evidências de estudos sobre o perfil de usuários atendidos, assim como da avaliação do atendimento odontológico na Clínica-escola da UFCG, Campus Patos-PB. A avaliação dos serviços de saúde na visão do paciente deveria ser um trabalho rotineiro na prática clinica pois sua análise permite obter um diagnóstico da realidade e partir daí desencadear movimentos de transformação na busca pela melhoria do atendimento. 
Sugere-se outros estudos abordando a mesma temática porém com foco nos alunos e professores quanto a confiança, atualização, competência, humanização, presteza, pontualidade, cordialidade, preparo técnico cientifico e organização do ambiente de trabalho, com possíveis sugestões para melhorar a qualidade do atendimento ofertado. Assim como estudos após a implantação das novas diretrizes curriculares dos cursos de odontologia que estabelecem como perfil de egresso de graduação, dentre outras características, um profissional generalista, dotado de sólida fundamentação técnico-científica e ativo, atento as necessidades individuais e coletivas, humanístico, comunicativo, capaz de se expressar com clareza e atuante na prática odontológica. Esses estudos permitirão avaliar alunos e o curso de odontologia quanto ao aspecto da clínica-escola, uma vez que as novas DCN's em seu capítulo VI estabelecem que deverão ser monitorados e permanentemente avaliados, os cursos de odontologia com o objetivo de acompanhar e permitir os ajustes necessários para seu aperfeiçoamento, e os estudantes, com base nas competências desenvolvidas visando o benefício dos indivíduos e da comunidade em que atua (Brasil, 2021).

\section{Conclusão}

Os pacientes da Clínica-escola de Odontologia da UFCG apresentaram um alto grau de satisfação em todas as dimensões avaliadas, sendo o intervalo de marcação entre as consultas, o aspecto que mais apresentou respostas negativas. Esses dados indicam que o serviço oferecido vai além dos conhecimentos técnicos adquiridos pelos acadêmicos na universidade, pois os discentes atuam de forma generalista e humanista seguindo os princípios éticos no processo saúde doença e resolvem de forma satisfatória e com qualidade as principais necessidades de saúde bucal que acometem seus usuários.

\section{Agradecimentos}

O presente trabalho foi realizado com apoio do CNPq, Conselho Nacional de Desenvolvimento Científico e Tecnológico - Brasil, por meio do programa Institucional de Bolsas de Iniciação Científica (PIBIC/CNPq-UFCG).

\section{Referências}

Azevedo, L. C. S. \& Rocha, M. P. (2018). Satisfação dos usuários de serviços públicos odontológicos: Revisão da literatura. Id on Line Revista Multidisciplinar e de Psicologia, 12(42), 704-707.

Bordin, D., Fadel, C. B., Moimaz, S. A. S., Garbin, C. A. S., \& Saliba, N. A. (2017). Estudo comparativo da satisfação de usuários e profissionais da saúde com o serviço público odontológico. Ciência \& Saúde Coletiva, 22(1), 151-160.

Borges, R. C., Otoni, T. A. C., \& Pires, R. C. C. P. (2015) Avaliação da qualidade do serviço odontológico prestado pela Faculdade de Odontologia da Universidade de Itaúna, MG, Brasil: visão do usuário, RFO UPF, 20(3), 308-312.

Brasil, Ministério da Educação. Conselho Nacional de Educação. Câmara de Educação Superior. Resolução CNE/CES nº $3 / 2021$ de 21 de junho de 2021. Diretrizes curriculares nacionais dos cursos de graduação em odontologia. Diário Oficial, Brasília, DF, 2021. Seção 1, p.77.

Brás, A. H., Corrêa, L. G., \& Eid, N. L. M. (2015). A avaliação da satisfação dos usuários da clínica odontológica do itpac-porto sobre a qualidade do atendimento prestado por acadêmicos do curso de odontologia. Revista da Academia Brasileira de Odontologia - AcBO. 4(2).

Brunhauser, A.L., Magro, M. L., \& Neves, M. (2013). Avaliação de serviço de saúde bucal: Um estudo comparativo. RFO UPF, 18(1), 24-31.

Donabedian, A. “Os sete pilares da qualidade”. (1990). Arch Pathol Lab Med, 114(11), 1115-1118.

Fernandes, L. M. A. G. (2002). Validação de um instrumento para a avaliação da satisfação dos usuários, com os serviços públicos de saúde bucal - QASSaB. [Tese]. Camaragibe (PE): Faculdade de Odontologia de Pernambuco.

Kitamura, E. S., Bastos, R. R., Palma, P. V., \& Leite, I. C. G. (2016). Avaliação da satisfação dos usuários dos Centros de Especialidades Odontológicas da macrorregião Sudeste de Minas Gerais. Epidemiol. Serv. Saúde, 25(1), 137-148.

Magalhães, B. G., Oliveira, R. S., Góes, P .S. A., \& Figueiredo, N. (2015). Avaliação da qualidade dos serviços prestados pelos Centros de Especialidade Odontológicas: visão dos usuários. Cad de Saúde Coletiva, 23(1), 76-85.

Marafon A. (2017). Avaliação da qualidade do atendimento na clínica integrada do curso de odontologia de uma ies de porto velho/ro: visão do usuário. [monografia] Porto Velho: Centro Universitário São Lucas.

Martins, R. J., Balila, N. M., Garbin, C. A. S., \& Garbin, A. J. I. (2017). Medo e ansiedade dos estudantes de diferentes classes sociais ao tratamento odontológico. Arch Health Invest, 6(1), 43-47. 
Melonardino, A. P., Rosa, D. P., \& Gimenes, M. (2016). Ansiedade: Detecção e conduta em odontologia. Rev UNINGÁ, 48, 76-83.

Mialhe, L. F., Gonçalo, C., \& Carvalho, L. M. S. (2008). Avaliação dos usuários sobre a qualidade do serviço odontológico prestado por graduandos do curso de Odontologia da FOP/Unicamp. RFO, 13(1):19-24.

Moimaz, S. A. S., Lima, A. M. C., Garbins, C. A. S., Corrente, J. E., \& Saliba, N. A. (2016). Avaliação da qualidade do atendimento na clínica integrada do curso de odontologia de uma ies de Porto Velho/RO: visão do usuário. Ciência \& Saúde Coletiva, 21(12), 3879-3887.

Nascimento, A. D. A., Silva, H. N. P., Brito, O. F. F., \& Barreira, A. K. (2020). Satisfação dos usuários atendidos na Clínica Integral de Odontologia da Universidade Federal de Pernambuco e fatores associados. RFO UPF, 25(1), 66-73.

Oliveira, R. O., Martins, E. P., Santana, J. L. B., Bezerra, S. R. S., \& Dourado, A. T. (2012) Avaliação dos usuários sobre a qualidade do atendimento odontológico prestado por alunos de odontologia. RFO UPF, 17(3), 319-325.

Pêgo, S. P. B et al. (2016). Avaliação dos serviços odontológicos prestados por acadêmicos: percepção do usuário. Rev Intercâmbio, 7, $138-147$.

Praxedes, C. S., Carneiro, S. V., Martins, L. F. B., Silva, C. H. F., Leite, A. C. R. M. (2017). Nível de satisfação dos pacientes atendidos no complexo odontológico do centro universitário católico de Quixadá - CE. Rev Expres Catól Saúde, 2(1):42-8.

Rezende, M. C. R. A., Lopes, M. R. A. N. E., Gonçalves, D. A., Zavanelli, A. C., \& Farjado, R. S. (2015). Acolhimento e bem estar no atendimento odontológico humanizado: o papel da empatia. Arch Health Invest, 4(3), 57-61.

Santos, L. M., Noro, L. R. A., Roncalli, A. G., \& Teixeira, A. K. M. (2016). Autopercepção sobre saúde bucal e sua relação com utilização de serviços e prevalência de dor de dente. Rev Ciência Plural, 2(2), 14-27.

Sousa, C. N. D., Souza, T. C. D., \& Araújo, T. L. C. D. (2015). Avaliação da satisfação dos pacientes atendidos na clínica escola de odontologia em uma instituição de ensino superior. Revista Interface, 3(8), 01-05.

Souza, P. G., Silva, M. B., Braga A. T., Siqueira, T. P., Gonçalves, L. C., \& Soares, P. V. (2014). Avaliação da qualidade do atendimento oferecido na Clínica Integrada da Universidade Federal de Uberlândia. Revista Odontol Bras Central. 23(66):140-45.

Torres, S.C., \& Costa I.C.C. (2015). Satisfação dos usuários atendidos nas clínicas integradas do departamento de odontologia da UFRN. Rev Ciência Plural, $1(2), 4-18$. 